\title{
The High Level Trigger of the CMS experiment
}

\author{
Xuyang Gao (on behalf of the CMS Collaboration)* \\ Beihang University (CN) \\ E-mail: gaoxyabuaa.edu.cn
}

The CMS experiment has been designed with a 2-level trigger system, the Level 1 Trigger (L1), implemented on custom-designed electronics, and the High Level Trigger (HLT), a streamlined version of the CMS off-line reconstruction software running on a computer farm. We will present the performance with the specific algorithms developed to cope with the increasing LHC pileup and bunch crossing rate using $13 \mathrm{TeV}$ data during 2015, and prospects for improvements brought to both L1 and HLT strategies to meet the new challenges for 2016 scenarios with a peak instantaneous luminosity of $1.2 \times 10^{34} \mathrm{~cm}^{-2} \mathrm{~s}^{-1}$ and 30 pileup events.

Fourth Annual Large Hadron Collider Physics

13-18 June 2016

Lund, Sweden

${ }^{*}$ Speaker. 


\section{The CMS Trigger}

The role of the trigger in a High Energy Physics experiment is to reduce the rate of recorded collision events to a level which is manageable by the following Data Acquisition (DAQ) and Reconstruction steps. The CMS experiment [1] has been designed with a 2-level trigger system, the Level 1 Trigger (L1), implemented on custom-designed electronics, and the High Level Trigger (HLT), a streamlined version of the CMS off-line reconstruction software running on a computer farm. At LHC the proton beams are organized in bunches. Those bunches were interleaved by 50 ns during the Run1 period and 25 ns at Run2.

With the beginning of the LHC Run 2, the CMS experiment has to cope with harsher operating conditions: higher center-of-mass energy of $13 \mathrm{TeV}$, leading to an enhancement of the production cross section of interesting processes like weak boson and top quark production, and larger occurrence of multiple proton-proton interactions in the same / near bunch crossings (pile-up), ranging from 20 to 40 simultaneous interactions. The HLT software has been optimized for these conditions, balancing a high selection efficiency on signal events while keeping the output rate at acceptable levels both for the online data acquisition system and the offline prompt reconstruction [2].

\section{Improvements in the HLT for Run 2}

There are several improvements in the HLT algorithms and menu prepared for Run2. Overall, the most effective one is the move of basically all HLT paths in CMS to Particle Flow based methods and event reconstruction. Particle Flow (PF) is a reconstruction technique widely used in CMS analyses. It makes use of the full detector information to describe the global collision event, by identifying particles individually and clustering them into more complex objects. Its use in HLT was already started during Run1, for tau leptons, jets and missing energy reconstructions [3].

\subsection{Isolated muons}

For muon candidates a new approach to isolation has been developed for Run 2, based on Particle Flow clusters (exploring the whole detector granularity) and sequential isolation (having separate isolation definitions for ECAL, HCAL and the tracker). Those improvements allow to maximize the selection efficiency for isolated muons while keeping similar kinematic thresholds as those used at the end of Run 1. Figure 1 shows the efficiency of the muon isolation in the ECAL and HCAL calorimeters. 

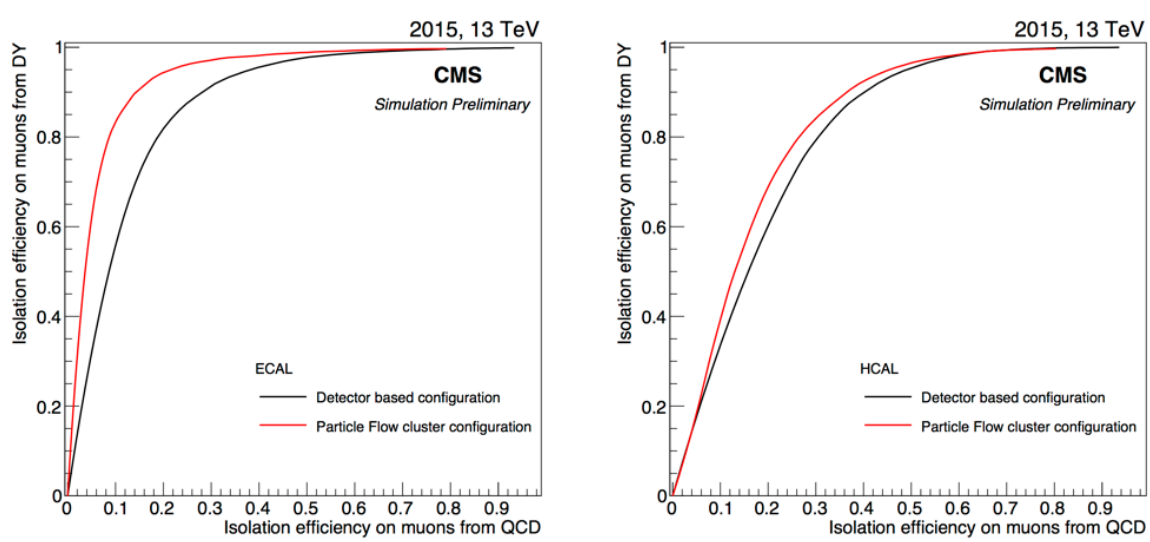

Figure 1: Performance of the ECAL (HCAL) calorimeter isolation are presented in left (right), for $Z \rightarrow \mu \mu$ signal events vs multi-jet background events, for an average pile-up of 40 and bunch spacing of $25 \mathrm{~ns}$.

\subsection{Isolated electrons}

For the electron case, CMS employed isolation techniques to allow for lower kinematic thresholds on the trigger algorithms. The isolation information used in the electron triggers comes from both Particle Flow clusters and reconstructed tracks. The improvements in the isolation allows the $P_{T}$ threshold of the single electron trigger to go as low as $23 \mathrm{GeV}$, and therefore maintain the physics potential for electroweak, top and Higgs processes. Figure 2 shows the distribution of the hadronic energy (left), relative HCAL isolation (middle), relative ECAL isolation (right).
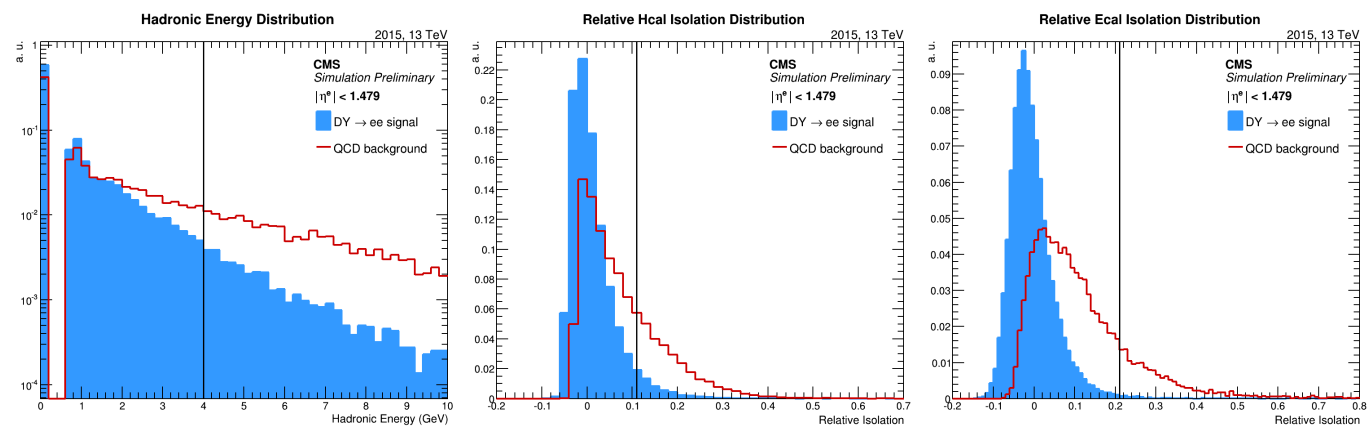

Figure 2: Distribution of the identification variables for events from Drell-Yan processes (blue full histograms) and for multi-jet background processes (red histograms).

\section{Performance in the HLT at Run 2}

The updated configuration of the HLT was successfully deployed during the 2015 run [4], allowing CMS to record data equivalent to approximately $3.6 \mathrm{fb}^{-1}$ of proton-proton collisions at 13 $\mathrm{TeV}$. As a result of the improvements to the HLT software, CMS was able to keep the kinematic object thresholds close to those used during Run 1. Figure 3, 4 show performance of some triggers. 

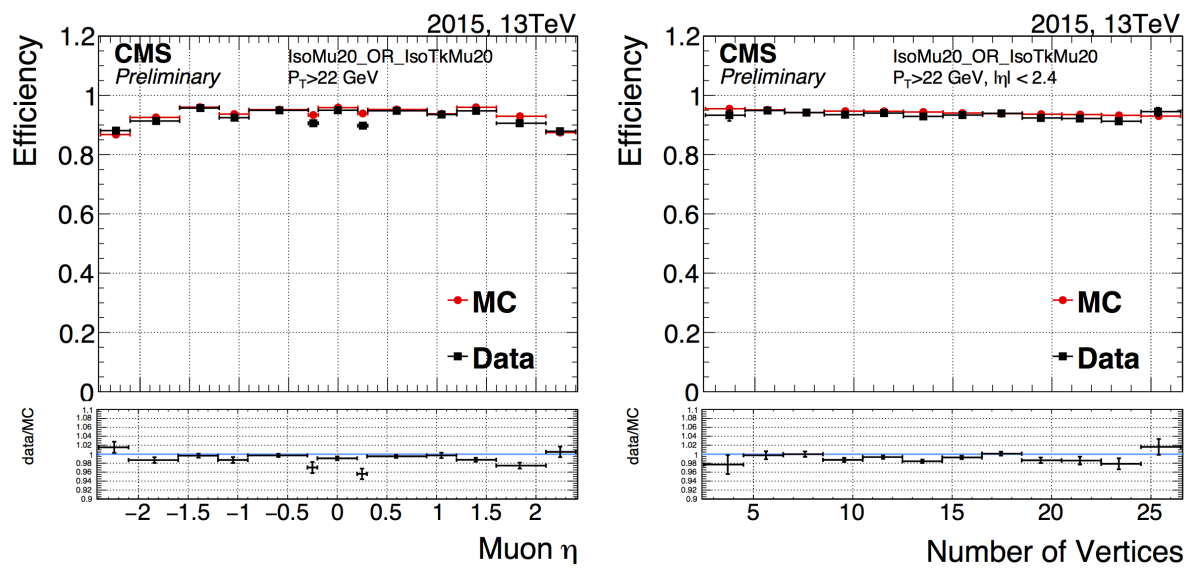

Figure 3: Efficiency as a function of $\eta$ (left) and Number of vertices (right) for the OR of the HLT_IsoMu20 and HLT_IsoTkMu20 with respect to the offline reconstructed muon passing identification and isolation requirements. These two trigger paths require a muon reconstructed online with $P_{T}>20 \mathrm{GeV}$.
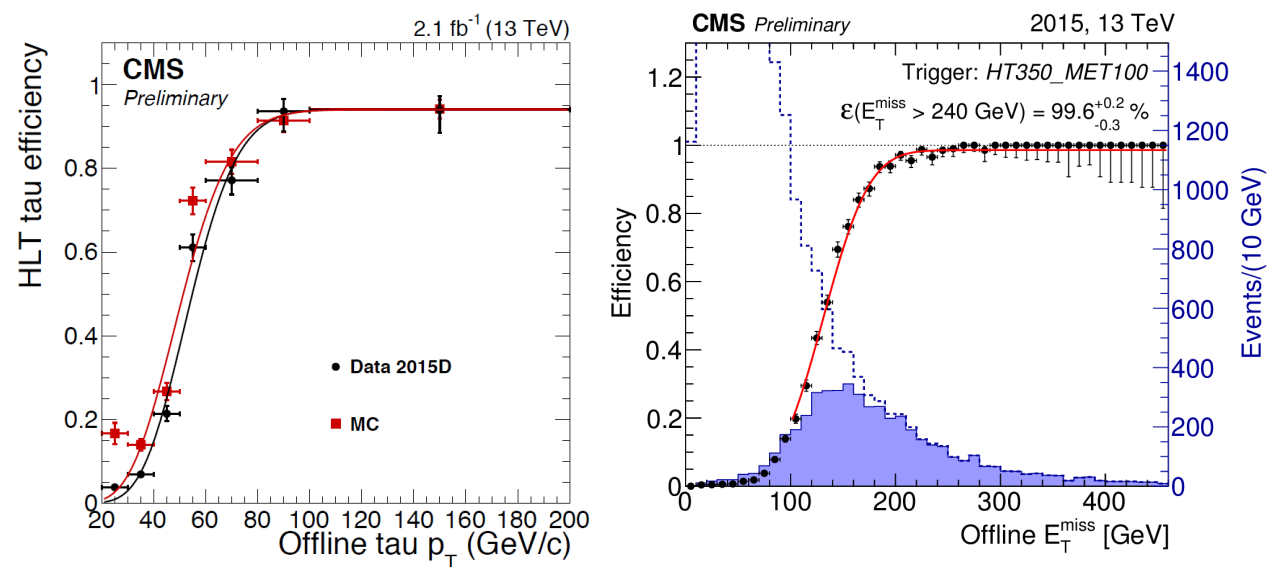

Figure 4: Left plot presents High Level Trigger efficiency of the $\tau_{h}$ leg of the $\tau_{h}+E_{T}^{\text {miss }}$ (medium isolation, $P_{T}>50 \mathrm{GeV}$, seeded by $E_{T}^{\text {miss }}$ Level-1) trigger for the $H^{ \pm} \rightarrow \tau_{h} v_{\tau}$ analysis. The Right plot presents the efficiency of the HLT_PFHT350_PFMET100 trigger as a function of offline $E_{T}^{\text {miss }}$, measured in $2.2 \mathrm{fb}-1$ of $13 \mathrm{TeV}$ data. The dashed histogram gives the number of total events, while the filled histogram gives the number of passed events. The dots with error bars give the efficiency.

\section{References}

[1] The CMS Collaboration, The CMS experiment at the CERN LHC, JINST 3:S08004, 2008.

[2] Andrea Perrotta, "Performance of the CMS High Level Trigger", CMS CR -2015/096.

[3] Thiago Tomei Fernandez, "Performance of electron, photon and muon triggers at the CMS High Level Trigger", CMS CR -2015/300.

[4] https:

//twiki.cern.ch/twiki/bin/view/CMSPublic/L1TriggerDPGResults\#c. 